\title{
Letter
}

\section{Multiple-Stage Converter Topology for High-Precision High-Current Pulsed Sources}

\author{
Nicolás Wassinger, Student Member, IEEE, Sebastián Maestri, Rogelio Garcia Retegui, Jean Marc Cravero, \\ Mario Benedetti, and Daniel Carrica, Senior Member, IEEE
}

\begin{abstract}
A new high-current, low-rise-time, and high-precision pulse generator is presented. The topology is based on the use of different stages, each one specific for a particular operation range in terms of power and switching frequency. This approach allows to accomplish current, voltage, and precision requirements with standard semiconductors. Moreover, the proposed topology provides an independent and flexible adjustment of the pulse parameters (rise and fall times, flat-top duration, pulse amplitude, etc.). Experimental results are provided to validate the control of the proposed topology.
\end{abstract}

Index Terms-Accelerator power supplies, current control, pulse power system.

\section{INTRODUCTION}

$\mathbf{H}$ IGH current pulsed power converters based on a capacitor-discharge topology are the best option for an inductive load that requires fast rise time, short settling time, and extremely precise flat top [1], [2]. This topology is composed by a capacitor bank that is initially charged to be later discharged through a semiconductor switch in the inductive load [3]. The capacitor bank and the load compose a damped resonant circuit. This basic circuit can be further improved with a third harmonic circuit and an active filter to produce high current pulses with a high precision flat top [4]. However, this system presents some disadvantages.

1) The current-pulse parameters (flat-top duration, and rise and fall times) depend on the resonant circuit and the input voltage.

2) There is a strong dependency on load parameters.

3) Reduced efficiency. Power dissipation circuits are often required to discharge the capacitor bank between pulses.

Manuscript received December 23, 2009; revised April 5, 2010, June 17 2010; accepted July 25, 2010. Date of current version June 22, 2011. This work was supported by the Universidad Nacional de Mar del Plata, Argentina, by the Consejo Nacional de Investigaciones Científicas y Técnicas (CONICET), Argentina, and by the Ministerio de Ciencia, Tecnología e Innovación Productiva (MINCYT), Argentina. Recommended for publication by Associate Editor B. Tamyurek.

N. Wassinger, S. Maestri, R. Garcia Retegui, M. Benedetti, and D. Carrica are with the Laboratorio de Instrumentación y Control, Universidad Nacional de Mar del Plata, Argentina (e-mail: nwassinger@fi.mdp.edu.ar; somaestri@ fi.mdp.edu.ar; rgarcia@fi.mdp.edu.ar; mbenedet@fi.mdp.edu.ar; carrica@fi. mdp.edu.ar).

J. M. Cravero is with the TE-EPC, European Organization for Nuclear Research, Geneva 23, Switzerland (e-mail: jean-marc.cravero@cern.ch).

Digital Object Identifier 10.1109/TPEL.2010.2064792

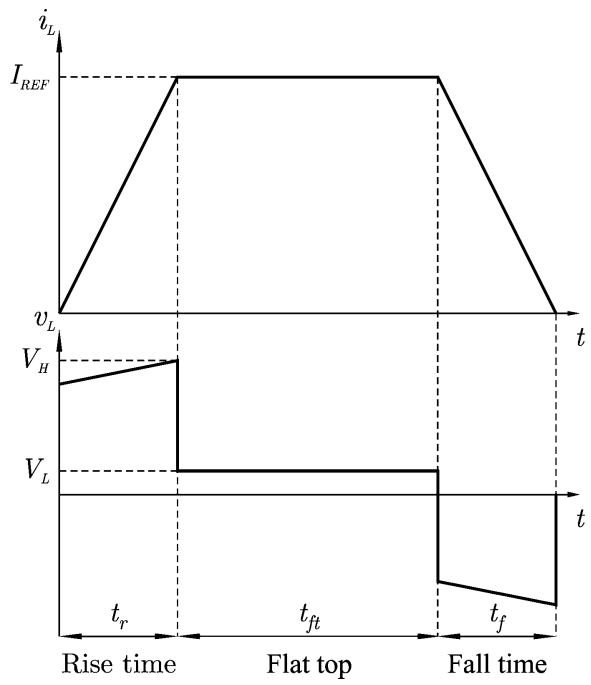

Fig. 1. Trapezoidal load-current waveform and corresponding applied voltage.

4) High rms value of the current pulse. The sinusoidal shape in the load current increases the rms value.

In order to overcome these drawbacks, this study proposes a novel topology for high current high precision pulsed current source applications.

\section{Multiple-Stage Converter Topology}

In pulsed power applications with an $R L$ load, a trapezoidal waveform is the best solution to reduce the load rms current. Fig. 1 shows the load current and voltage. The current waveform implies the application of a high voltage $V_{H}$ during rise and fall times, and a low voltage $V_{F T}$ during the flat top.

The necessary input voltage to reach a current flat top $I_{\mathrm{REF}}$ in a rise time $t_{r}$ can be calculated as:

$$
V_{H}=\frac{I_{\mathrm{REF}} \cdot L}{t_{r}}+I_{\mathrm{REF}} \cdot R \approx \frac{I_{\mathrm{REF}} \cdot L}{t_{r}}
$$

where the load resistance has been considered negligible, due to the low influence of this term in typical pulsed loads. The flat-top voltage is given by the drop voltage on the resistive component of the load $\left(V_{L}=I_{\mathrm{REF}} \cdot R\right)$.

The development of semiconductor devices with increased current and voltage capabilities offers new possibilities to develop converters with improved performances [5]-[7]. However, the semiconductor technology does not allow to obtain 


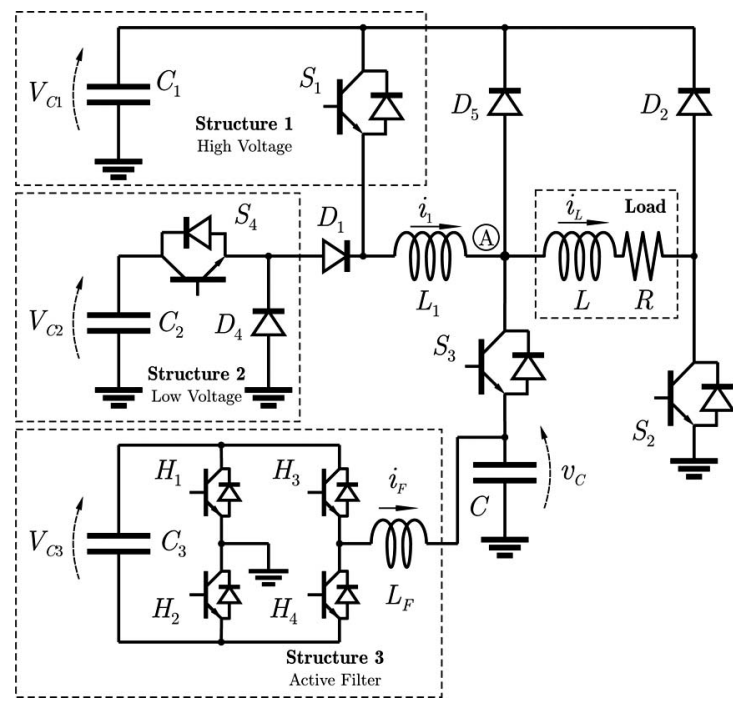

Fig. 2. Simplified schematic of the proposed topology.

with a same component the high currents (few kiloamperes), the high voltages (several kilovolts) and the high switching frequency necessary to achieve the high precision required. A twostage voltage converter based on a high-voltage stage for the rise and fall times and a low voltage stage to control the flat-top current is proposed in [8]. This approach allows to reach the flat-top current in a shorter time and to decrease the switching frequency at flat top compared to a single-stage converter. However, since the flat-top structure must handle the total output current, the precision is bounded by the maximum switching frequency of its high-current semiconductors.

To overcome these problems a multiple stage current converter based on three structures with different voltage and current ranges that connect sequentially during the pulse-generation stages is proposed (see Fig. 2). The aim is to use structure 1 (high voltage and current) during rise and fall times, structure 2 (low voltage and high current) to control the flat-top mean current with moderate precision, and structure 3 (low voltage and current) to control the load current with the required precision.

The operational principle of this topology is summarized as follows.

Rise time: During this stage, the only structure that remains active is structure 1 , which initiates the charge of $L_{1}$ and $L$ through the high voltage source $V_{C 1}=I_{\mathrm{REF}} \cdot\left(L+L_{1}\right) / t_{r}$. Therefore, switches $S_{1}$ and $S_{2}$ are turned ON and $S_{3}$ and $S_{4}$ are turned OFF. If the load resistance is negligible, $i_{1}=i_{L}$ increase with a slope given by $V_{C 1} /\left(L_{1}+L\right)$.

Flat top: When currents $i_{L}=i_{1}$ reach the reference value $I_{\mathrm{REF}}$, structure 1 is disconnected and structures 2 and 3 are connected by means of $S_{1}$ turning-off and $S_{3}$ turning-on. The voltages of these structures, $V_{C 2}$ and $V_{C 3}$, must be higher than $I_{\mathrm{REF}} \cdot R$. The turning-on of $S_{3}$ allows the connection of the active filter and the capacitor $C$ to the node (A). $C$ is connected in parallel to structure 3 to avoid overvoltage in this node.

Since structure 2 must handle the high load current, a medium $S_{4}$ switching frequency, $f_{I 1}$, must be adopted. As a consequence, a peak-to-peak ripple current $\Delta I_{1}$, much higher than
TABLE I

SEMICONDUCTOR DEVICES REQUIREMENTS

\begin{tabular}{l|c|c|c}
\hline Devices & Voltage & Current & Operation frequency \\
\hline$S_{1}, S_{2}, D_{1}, D_{2}$ & High $\left(V_{C 1}\right)$ & High $\left(I_{R E F}\right)$ & Very low $\left(f_{P}\right)$ \\
$S_{3}, D_{5}$ & High $\left(V_{C 1}\right)$ & Low $\left(\Delta I_{1}\right)$ & Very low $\left(f_{P}\right)$ \\
$S_{4}, D_{4}$ & Low $\left(V_{C 2}\right)$ & High $\left(I_{R E F}\right)$ & Medium $\left(f_{I 1}\right)$ \\
$H_{1} \cdots H_{4}$ & Low $\left(V_{C 3}\right)$ & Low $\left(\sim \Delta I_{1}\right)$ & High $\left(f_{I F}\right)$ \\
\hline
\end{tabular}

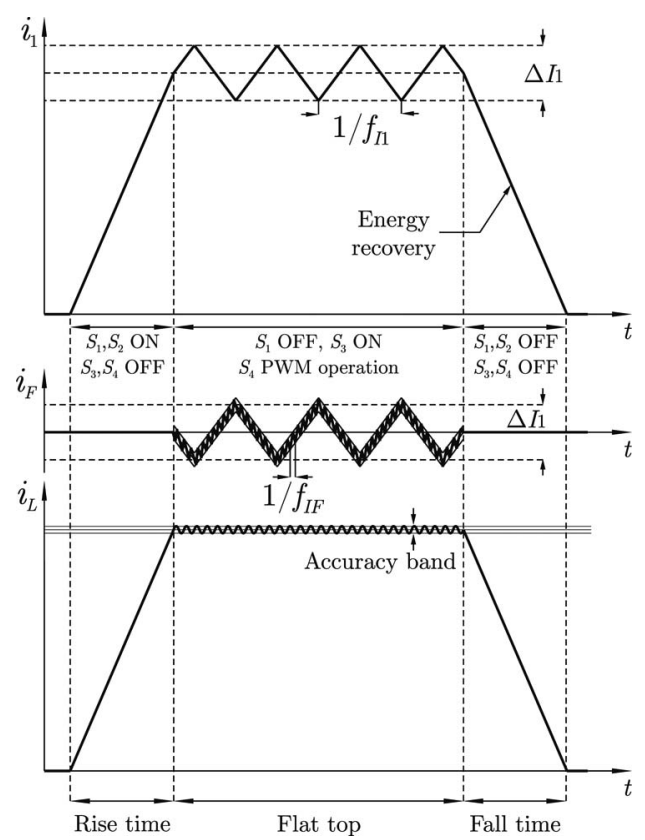

Fig. 3. Current waveforms and operation principle.

the required one, is obtained. Assuming that the forward voltage of the devices is negligible, the expression of $\Delta I_{1}$ is given by

$$
\Delta I_{1}=\frac{v_{C}}{L_{1} \cdot f_{I 1}} \cdot\left(1-\frac{v_{C}}{V_{C 2}}\right) .
$$

In order to cancel $\Delta I_{1}$, structure 3 must operate at a switching frequency $f_{\mathrm{IF}}$ higher than $f_{I 1}$. This structure generates a ripple current $\Delta I_{f}$ as follows:

$$
\Delta I_{f}=\frac{V_{C 3}}{2 \cdot L_{f} \cdot f_{\mathrm{IF}}} \cdot\left(1-\frac{v_{C}^{2}}{V_{C 3}^{2}}\right) .
$$

Fall time: To decrease the load current, all switches are turned OFF. The energy stored in the load and inductor $L_{1}$ returns to the capacitor bank $C_{1}$ through $D_{1}, D_{2}$, and $D_{4}$. The current difference between $i_{L 1}$ and $i_{L}$ when $S_{3}$ is turned OFF flows through $D_{5}$.

Table I resumes the requirements for the semiconductor devices, where $f_{P}$ is the pulse repetition frequency.

Fig. 3 shows the current waveforms of the proposed system and the state of the different semiconductor switches.

\section{CONTROL SYSTEM}

As the system is composed by different interconnected structures, a transient is generated each time a structure is 


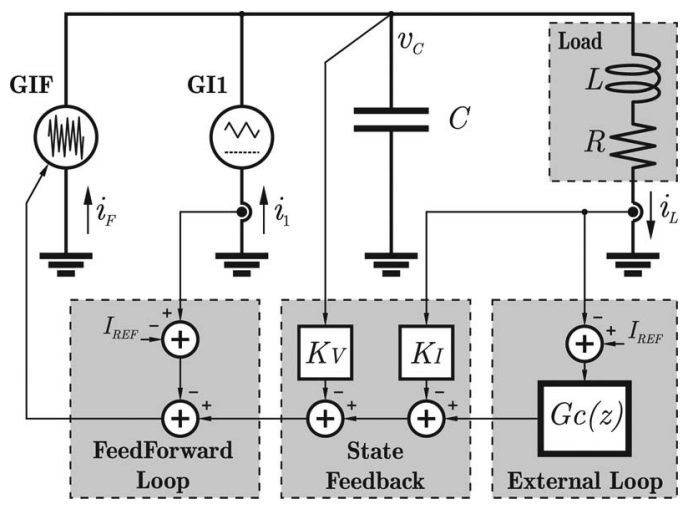

Fig. 4. Flat-top equivalent circuit.

connected/disconnected. This aspect is critical at the beginning of the flat top, as the generated transient can exceed the system specifications of duration and amplitude. Fig. 4 shows an equivalent circuit of the system in the flat top, where $G_{I 1}$ and $G_{\mathrm{IF}}$ current generators model $i_{1}$ and $i_{F}$, respectively.

The capacitor and the load form a second-order circuit with a low damping factor. If the initial conditions of the circuit are not appropriate $\left(i_{1}=i_{L} \neq I_{\mathrm{REF}}\right.$ or $\left.v_{C} \neq I_{\mathrm{REF}} \cdot R\right)$, an undesired oscillatory response will be generated. In practice, the appropriate conditions are very difficult to obtain due to possible delays in the devices operation (i.e., $S_{1}$ ) and measurement errors. Therefore, the control system must be able to handle the transient response. In order to achieve this, a state-feedback control is proposed to place the closed-loop poles of the secondorder circuit at $\omega_{P}$, so as to obtain a critical damped response. The feedback gains $K_{V}$ and $K_{I}$ are given by

$$
\begin{aligned}
K_{V} & =\frac{C}{K_{f}}\left(2 \cdot \omega_{P}-R / L\right) \\
K_{I} & =\frac{1}{K_{f}}\left(\frac{\omega_{P}^{2}}{\omega_{0}^{2}}-K_{f} \cdot K_{V} \cdot R-1\right)
\end{aligned}
$$

where $\omega_{0}$ is the resonant frequency of the $R L C$ circuit and $K_{f}$ is the active filter gain. This gain is assumed constant as the bandwidth of the active filter is much larger than the bandwidth of the system.

As the resulting transfer is a type- 0 system, there will be a steady-state error in the mean value of $i_{L}$, which is compensated by an external loop with an integral-type controller. The gain of this controller is adjusted to obtain a cutoff frequency of $\omega_{C}$. In order to obtain a transient response mainly dependant on the external loop bandwidth, the poles of the state feedback are placed above the bandwidth $\left(\omega_{P}>\omega_{C}\right)$.

The precision of the flat-top current depends mainly on the rejection of the system to current ripples $\Delta I_{f}$ and $\Delta I_{1}$. Regarding $\Delta I_{f}$, it can only be attenuated by the $R L C$ circuit transfer function (5), since the feedback loops do not reject it

$$
\frac{\Delta I_{L}}{\Delta I_{f}}=\frac{\omega_{0}^{2}}{s^{2}+2 \cdot \xi \cdot \omega_{0} \cdot s+\omega_{0}^{2}} \approx \frac{\omega_{0}^{2}}{\omega_{\mathrm{IF}}^{2}} .
$$

TABLE II

SEMICONDUCTOR DEVICES REQUIREMENTS

\begin{tabular}{l|c|c|c}
\hline Devices & Voltage & Current & Operation frequency \\
\hline$S_{1}, S_{2}, D_{1}, D_{2}$ & $V_{C 1}=3.3 \mathrm{kV}$ & $I_{R E F}=2 \mathrm{kA}$ & $f_{P}=1 \mathrm{~Hz}$ \\
$S_{3}, D_{5}$ & $V_{C 1}=3.3 \mathrm{kV}$ & $\Delta I_{1}=48 \mathrm{~A}$ & $f_{P}=1 \mathrm{~Hz}$ \\
$S_{4}, D_{4}$ & $V_{C 2}=500 \mathrm{~V}$ & $I_{R E F}=2 \mathrm{kA}$ & $f_{I 1}=5 \mathrm{kHz}$ \\
$H_{1} \cdots H_{4}$ & $V_{C 3}=500 \mathrm{~V}$ & $\sim \Delta I_{1}=48 \mathrm{~A}$ & $f_{I F}=100 \mathrm{kHz}$ \\
\hline
\end{tabular}

$\Delta I_{1}$ rejection depends on feedback loops and is given by

$$
\frac{\Delta I_{L}}{\Delta I_{1}}=\frac{1}{K_{f}} \cdot \frac{K /\left(\left(s / \omega_{P}\right)+1\right)^{2}}{1+\left(\omega_{C} / s\left(s / \omega_{P}+1\right)^{2}\right)}
$$

where $K=K_{f} /\left(1+K_{V} \cdot K_{f} \cdot R+K_{I} \cdot K_{f}\right)$ is the gain of the transfer function resulting from the state feedback of the $R L C$ circuit. The worst-case ripple rejection occurs when $\omega_{C}<$ $\omega_{I 1}<\omega_{P}$, then $\Delta I_{1}$ is attenuated as

$$
\frac{\Delta I_{L}}{\Delta I_{1}} \approx \frac{K}{K_{f}}=\frac{1}{1+K_{V} \cdot K_{f} \cdot R+K_{I} \cdot K_{f}} .
$$

Equation (7) shows that the ripple rejection on the flat top depends inversely on $R$. Hence, as the increase of the heating power dissipation tends to increase the load resistance, $\Delta I_{L}$ is reduced and the rejection is improved. Operating with (4) and (7) yields a simpler expression

$$
\frac{\Delta I_{L}}{\Delta I_{1}} \approx \frac{\omega_{0}^{2}}{\omega_{P}^{2}} .
$$

Equation (8) shows that a better rejection requires a $\operatorname{larger} \omega_{P}$, i.e., a larger active filter frequency. In order to avoid increasing the active filter frequency, a feedforward loop is incorporated. This compensation is obtained by adding $i_{1}-I_{\mathrm{REF}}$ to the external loop reference. In this case, $\Delta I_{1}$ in (8) is replaced by the residual ripple of the feedforward compensation, which allows to obtain a higher rejection.

\section{POWER SYSTEM}

To evaluate aspects related to the power implementation, a pulsed source with the following specifications was considered: $I_{\mathrm{REF}}=2 \mathrm{kA}, L=1 \mathrm{mH}, R=0.15 \Omega, t_{r}=t_{f}=1 \mathrm{~ms}, f_{P}=$ $1 \mathrm{~Hz}$, and flat-top precision of $5 \times 10^{-4}$. Using (1)-(3), the requirements given in Table II were obtained.

$f_{I 1}$ and $f_{\mathrm{IF}}$ were adopted to obtain a low stress on the semiconductors $S_{4}, D_{4}$, and $H_{1} \ldots H_{4}$, which is defined by the required lifetime as regards thermal cycling capability. Concerning $S_{1}, S_{2}, S_{3}, D_{1}, D_{2}$, and $D_{5}$, their stress will depend on $f_{P}$, which is very low.

The delays of $S_{4}$ and $H_{1} \ldots H_{4}$ generate an increase of $\Delta I_{1}$ and $\Delta I_{f}$ ripples. Hence, they must be considered when the ripple rejection is adjusted by (7) and (8).

The reverse-recovery time of $D_{4}$ produces a reverse current proportional to the diode forward current and its slope during turn off, which flows through $S_{4}$ in addition to $I_{1}$. Moreover, during this recovery time, both $S_{4}$ and $D_{4}$ are forward biased simultaneously. Hence, the $S_{4}$ current is only limited by stray inductances. Since $i_{D 4}$ forward current and its slope are high, the maximum value of the reverse current can damage $S_{4}$; therefore, 


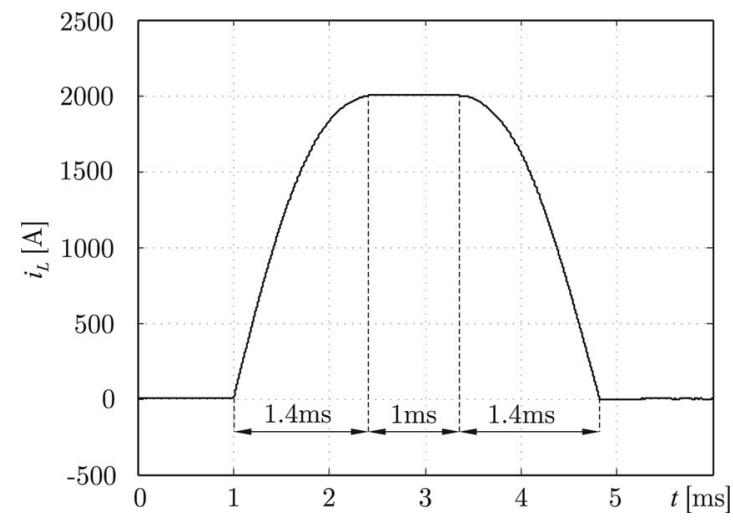

Fig. 5. Load current with capacitor-discharge converter.

it should be taken into account for $S_{4}$ ratings. Additionally, stray inductances could generate an overvoltage across the diode that can damage it. This effect can be solved by using a low reverse-recovery time diode with a conventional snubber circuit. Although this phenomenon impacts on the converter efficiency, it does not affect the precision of the flat-top current.

In the flat top, the most influential parasitic component is the capacitor $C$ equivalent series resistance, ESR, which modifies the poles and adds a zero at $\omega_{z}=1 /(\mathrm{ESR} \cdot C)$ of the $R L C$ function transfer. The poles modification is not a drawback, since they can be relocated by means of the feedback gains adjustment $\left(K_{V}\right.$ and $\left.K_{I}\right)$. On the other hand, the zero could decrease the $\Delta I_{f}$ ripple rejection calculated in (5), which affects the flat-top precision. To avoid this, the zero location must be $\omega_{z}>\omega_{\mathrm{IF}}$, which can be achieved by adopting a capacitor with an appropriate ESR or through a parallel array of capacitors.

Concerning $L_{1}$ and $L_{f}$, their possible variations (e.g., by hysteresis or by saturation) will change either the frequencies $f_{I 1}$ and $f_{\mathrm{IF}}$ (if hysteresis current control is used) or $\Delta I_{1}$ and $\Delta I_{f}$ (if fixed frequency current control is used). In both cases, the ripple rejection will be modified. Therefore, the lowest inductance values of $L_{1}$ and $L_{f}$ should be considered either to adjust the hysteresis bands or the ripple rejection by means of (5) and (8). Another characteristic of $L_{1}$ and $L_{f}$ is their series resistances, which are low. In general, as the switching times are much lower than the associated time constants, the current waveforms are practically linear.

\section{Simulations}

In order to compare the proposed topology with the capacitive discharge one, both converters have been evaluated using MATLAB/Simulink. The simulation integrates the different effects of digital control, like communication and calculation delays or $\mathrm{A} / \mathrm{D}$ conversions. The load and current pulse characteristics are the same as the ones in Section IV, considering a $t_{f t}$ of 1-3 ms.

Fig. 5 shows a current pulse obtained with the capacitordischarge converter for a $t_{f t}=1 \mathrm{~ms}$. The resonant frequency and the input voltage were adjusted to $133 \mathrm{~Hz}$ and $2914 \mathrm{~V}$, respectively. It can be noted that $t_{r}$ and $t_{f}$ are close to $1.4 \mathrm{~ms}$.

If a flat top of $3 \mathrm{~ms}$ is considered, the resonant frequency and charging voltage should be modified to $44 \mathrm{~Hz}$ and $980 \mathrm{~V}$. The

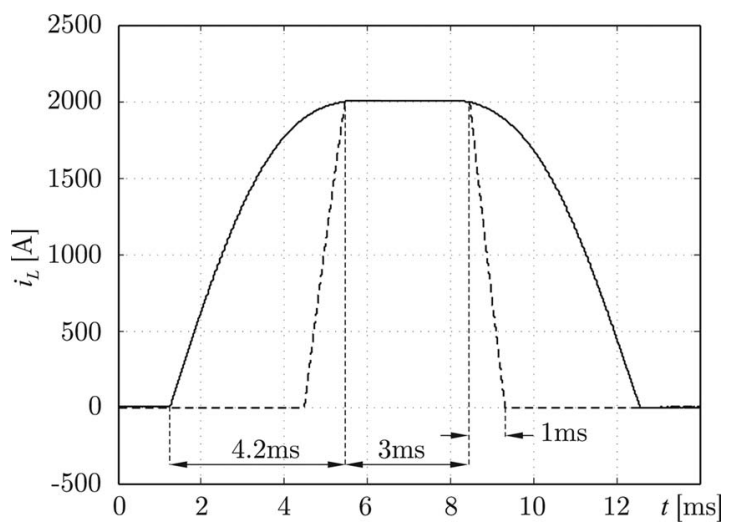

Fig. 6. Load current (solid line: capacitor-discharge converter, dashed line: proposed topology).

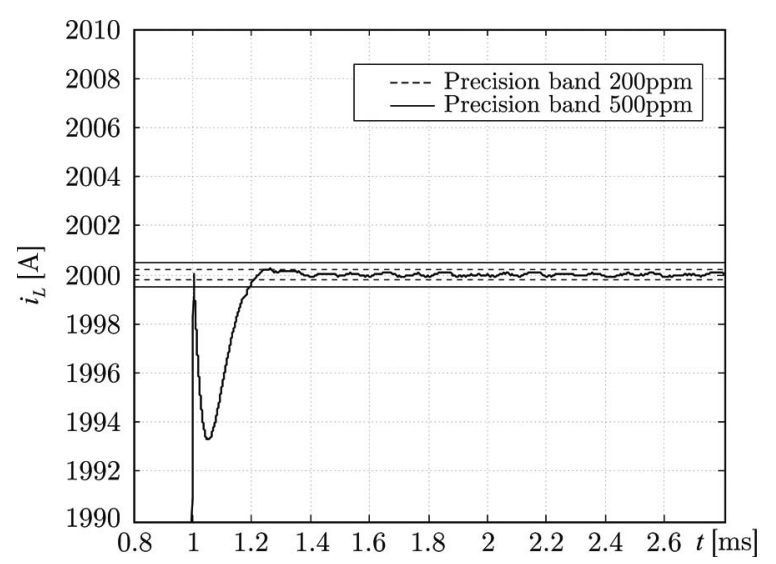

Fig. 7. Detail of the load current during flat top.

most critical-parameter modification is the resonance frequency as it involves modifying the circuit elements. Moreover, the decrease in the resonance frequency leads to higher rise and fall times, which increases the associated current effective value [see Fig. 6 (solid line)].

In the case of the proposed topology, the $t_{r}$ and $t_{f t}$ adjustments are independent and are defined by $V_{C 1}$ (1) and $C_{2}$, respectively. $C_{2}$ is sized in order to be able to deliver the required energy to the load (which is defined by $t_{f t}$ and $I_{\mathrm{REF}}$ ), with a voltage drop that does not affect the $i_{1}$ control, i.e., $V_{C 2 \min }>v_{C}$. In this case, $C_{2}$ is a $60 \mathrm{mF} / 500 \mathrm{~V}$ capacitor bank. Fig. 6 (dashed line) shows a current pulse of $t_{f t}=3$ ms using the proposed topology. It can be seen that, $t_{r}$ and $t_{f}$ are significantly lower, with a $30 \%$ reduction of the effective value.

Fig. 7 shows a zoom of the load current during flat top. The transitory response at the beginning of flat top is adjusted to $200 \mu$ s by taking $\omega_{C}=2 \pi 2.7 \mathrm{kHz}$. The bandwidth and precision of the sensors have been assumed adequate for a high-precision measurement, which allows to obtain a precision close to 200 ppm. Generally, the precision will depend on both the sensors and the semiconductors switching frequencies. 
TABLE III

PROTOTYPE PARAMETERS

\begin{tabular}{lc|lc}
\hline$L$ & $1 \mathrm{mH}$ & $V_{C 2}=V_{C 3}$ & $10 \mathrm{~V}$ \\
$R$ & $0.15 \Omega$ & $I_{R E F}$ & $15 \mathrm{~A}$ \\
$L_{1}$ & $500 \mu \mathrm{H}$ & $t_{r}, t_{f}$ & $1 \mathrm{~ms}$ \\
$L_{F}$ & $50 \mu \mathrm{H}$ & Settling time & $<200 \mu \mathrm{s}$ \\
$V_{C 1}$ & $25 \mathrm{~V}$ & Flat-top duration & $3 \mathrm{~ms}$ \\
\hline
\end{tabular}

\section{EXPERIMENTAL RESULTS}

Experimental tests were carried out on a low-power-scale prototype. The aim of these tests was to validate the controlled interconnection of stages and the associated control of the proposed topology, without focusing on the resulting precision. The current and voltage values of this prototype have been scaled down to laboratory levels, while the switching frequencies have been kept to maintain the system dynamics. The main parameters of the system are listed in Table III.

The current control of $i_{1}$ and $i_{F}$ is performed by means of hysteresis current control. The hysteresis bands of $i_{1}$ and $i_{F}$ are adjusted to obtain a maximum switching frequency of 5 and $100 \mathrm{kHz}$, respectively. In order to fulfil the settling time requirement, the external loop bandwidth was adjusted to $2.7 \mathrm{kHz}$, while the closed-loop poles of the state feedback were located in $15 \mathrm{KHz}$ so as not to affect the stability of the external loop or to influence the transient response.

The proposed control system was implemented by a specific electronic board composed of a Xilinx FPGA XCS40XL and a 16-bit analog/digital acquisition module. The main block of the control system performs the following tasks:

1) structure interconnection management;

2) $i_{1}$ and $i_{F}$ digital hysteresis current controls,

3) control loop algorithms,

4) management of control and communication with the ana$\log$ /digital acquisition module.

The acquisition module consists of four 16-bit ADC with their corresponding differential input filters, while the sampling frequency is $1 \mathrm{MHz}$. The measurement of $i_{1}$ and $i_{L}$ was performed with Hall effect current sensors (accuracy $\pm 0.5 \%$ ) and the measurement of $i_{F}$ through a Pearson-type current sensor (accuracy $\pm 1 \%$ ).

A current pulse with no significant transient response during the coupling of structures (beginning and end of the flat top) was obtained; times $t_{r}, t_{f}$, and $t_{f t}$ were within specifications (see Fig. 8). $t_{r}$ was greater than $t_{f}$ because, during the rise time, the forward voltage of the devices and $V_{C 1}$ have an opposite polarity, while during the fall time they have the same polarity. In the full-scale prototype, this effect will be negligible since $V_{C 1}$ is much greater than the voltage drop of the devices.

The transitory response at flat top beginning is shown in Fig. 9, where its duration is $184 \mu \mathrm{s}$ and its amplitude is $0.7 \%$ of $I_{\mathrm{REF}}$. The measured precision in the flat top is close to $500 \mathrm{ppm}$.

\section{CONCLUSION}

A new multiple-stage converter topology for pulsed current sources was proposed. The proposed topology allows to fulfil

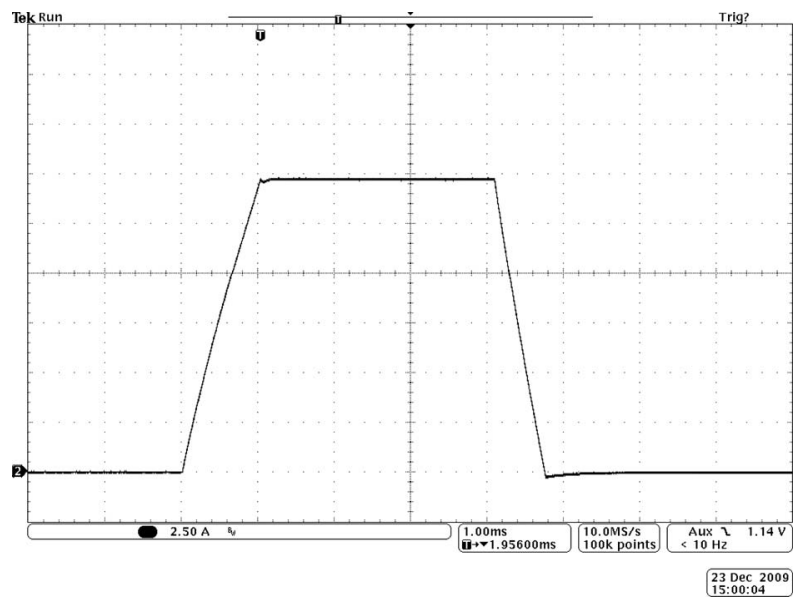

Fig. 8. Experimental results of the scaled-down prototype. Current pulse measurement (2.5 A/division, $1 \mathrm{~ms} /$ division).

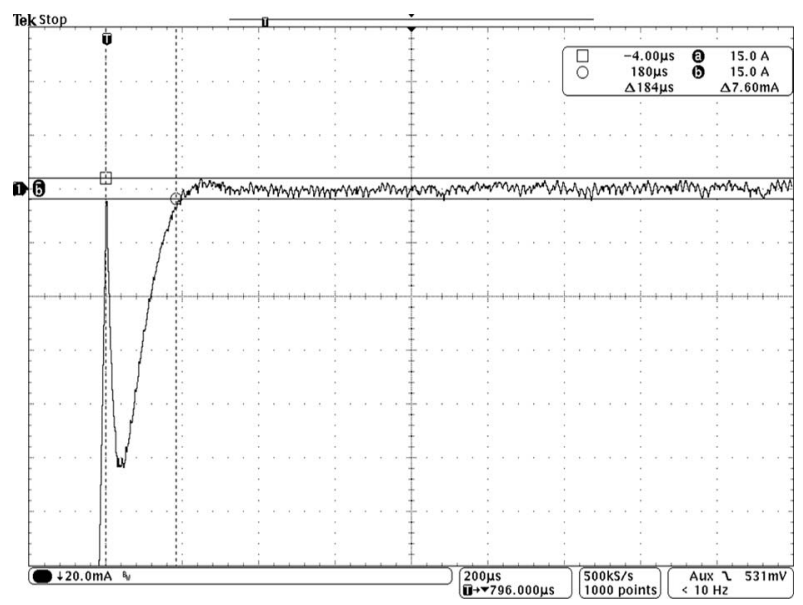

Fig. 9. Experimental results of the scaled-down prototype. Flat-top transient response ( $20 \mathrm{~mA} /$ division, $200 \mu \mathrm{s} /$ division $)$.

the requirements of flat top and rise/fall times independently. This feature provides flexibility in the adjustment of the pulse parameters, which allows the reduction of the pulse current effective value. Furthermore, the proposed system allows the recovery of the load energy during the fall time.

The aforementioned characteristics make the proposed topology suitable for high current pulses with high precision flat top and short rise and fall times applications. Besides, it allows to extend the converter operation boundaries parallel to the semiconductor technology improvements.

\section{REFERENCES}

[1] F. Voelker, "Pulsed capacitor discharge power converter: An introductory overview," presented at the CERN Accel., School: Power Converters Part. Accel. Conf., Montreux, Switzerland, Mar. 1990.

[2] J. Cravero, G. Maire, and J.-P. Royer, "High current capacitor discharge power converters for the magnetic lenses of a neutrino beam facility," in Proc. Eur. Conf. Power Electron. Appl., Sep., 2007, pp. 1-8.

[3] C. Hummer, H. Singh, and D. Piccone, "Thyristors for pulsed power applications," in Proc. 24th Int. Power Modulator Symp., Conf. Rec., Jun. 2000, pp. 78-80. 
[4] J. Royer, "High current with high precision flat-top capacitor discharge power septum magnets," presented at the CERN/PS 95-13 EP2 Forum 95, Electr. Power Technol. Eur. Phys. Res., Montreux, Switzerland, 1995.

[5] S. Scharnholz, R. Schneider, E. Spahn, A. Welleman, and S. Gekenidis, "Investigation of IGBT-devices for pulsed power applications," in Proc. 14th IEEE Int. Pulsed Power Conf. Dig. Tech. Pap., Jun. 15-18, 2003, vol. 1, pp. 349-352.

[6] N. Luther-King, E. M. S. Narayanan, L. Coulbeck, A. Crane, and R. Dudley, "Comparison of trench gate IGBT and CIGBT devices for increasing the power density from high power modules," IEEE Trans. Power Electron., vol. 25, no. 3, pp. 583-591, Mar. 2010.

[7] V. Zorngiebel, E. Spahn, G. Buderer, A. Welleman, and W. Fleischmann, "Compact high voltage igbt switch for pulsed power applications," IEEE Trans. Magn., vol. 45, no. 1, pp. 531-535, Jan. 2009.

[8] E. Dallago, G. Venchi, S. Rossi, M. Pullia, T. Fowler, and U. Nielsen, "The power supply for a medical synchrotron beam chopper system," in Proc. 34th Annu. Conf. IEEE Ind. Electron. (IECON), 2008, pp. 10161020. 